\title{
Epithelioid Hemangioendothelioma of the Foot
}

\author{
Christopher Sardon ${ }^{1 *}$, Christin Foster ${ }^{2}$, Javier De Luca-Johnson ${ }^{3}$, Adam Fehringer ${ }^{1}$ \\ 1. Department of Radiology, Naval Medical Center Portsmouth, Portsmouth, USA \\ 2. Department of Radiology, Dartmouth-Hitchcock Medical Center, Lebanon, USA \\ 3. Department of Pathology, Naval Medical Center Portsmouth, Portsmouth, USA \\ * Correspondence: Christopher Sardon, Attn: Radiology, Naval Medical Center Portsmouth, 620 John Paul Jones Cir., Portsmouth, VA \\ 23708, USA \\ (入sardonc@gmail.com)
}

Radiology Case. 2021 Dec; 15(12):20-28 :: $\quad$ DOI: 10.3941/jrcr.v15i12.4052

\begin{abstract}
We describe the case of an 18-year-old male Army reservist presenting with left lower extremity pain for which he was initially diagnosed with a stress injury. After failing conservative treatment, a radiograph was obtained showing a "lacelike" appearance of the medullary bone in the foot and ankle. Magnetic resonance imaging subsequently demonstrated widespread polyostotic marrow replacement with coarsened trabeculations. A biopsy was obtained which diagnosed the patient with polyostotic epithelioid hemangioendothelioma which is the most common malignant vascular tumor of bone. The patient ultimately underwent a below the knee amputation once computed tomography of the chest, abdomen, and pelvis excluded distant metastatic disease. It is important for radiologists to be aware of this diagnosis because osseous epithelioid hemangioendothelioma can present like a stress injury and be mistaken for a less serious diagnosis while potentially having visceral involvement.
\end{abstract}

\section{CASE REPORT}

\section{CASE REPORT}

An 18-year-old male Army reservist presented with left lower extremity pain for one year. The pain began after a ruck march during basic training at which time he was diagnosed with a stress injury. His pain stopped during a 9-month period away from military operations and restarted when he began running again. The pain was aggravated by use and partially relieved by rest.

Initial radiographs demonstrated a diffuse "lacelike" appearance of medullary bone with mixed areas of prominent trabeculation interspersed with lucent areas involving the distal tibia and bones of the foot (Fig. 1). Subsequently, nuclear medicine bone scintigraphy was performed which showed moderate generalized uptake of the left ankle, mid tarsal region and first ray with focal uptake in the left 5th metatarsal. These findings were suggestive of a chronic, infiltrative bone marrow process for which magnetic resonance imaging (MRI) was recommended. The differential diagnosis considered at this time included sarcoidosis, infiltrative bone marrow disorders, hyperparathyroidism, multiple enchondromas, and intraosseous hemangiomas.

An MRI with and without contrast of the left foot and ankle demonstrated widespread polyostotic marrow replacement with preserved, coarsened trabeculations. The marrow signal abnormality was isointense to muscle on T1-weighted imaging (Fig. 3A), slightly hyperintense to muscle on $\mathrm{T} 2$-weighted imaging (Fig. 3B) and showed uniform enhancement following intravenous contrast (Fig. 3C). Surrounding the marrow abnormalities were lobular, thin sclerotic margins. Additionally, there were areas of high-grade endosteal scalloping and a small focus of intense synovial enhancement 
adjacent to the lateral talar dome. The primary differential diagnosis at this time included sarcoidosis and fibrovascular replacement of bone marrow. Alternatively, uncommon presentations of more common processes were also considered although thought less likely. These included: low-grade chondral lesions, aneurysmal bone cyst, tuberculosis, leukemia, lymphoma, and metastases. Since a definitive diagnosis was unable to be made radiographically, a biopsy was recommended.

Open biopsy and curettage were performed of the distal lateral tibia. This location was selected because it is easily accessible and would have less morbidity than the smaller bones of the foot and ankle. The biopsy sections submitted demonstrated sheets of large epithelioid cells infiltrating the marrow space and eliciting a reactive woven bone response (Fig. 4A). The epithelioid cells were characterized by abundant eosinophilic cytoplasm and oval to kidney-shaped nuclei with fine chromatin. Occasional intracytoplasmic vacuolization was identified, with some of the vacuoles filled with red blood cells (Fig. 4B and 4C). Cytologic atypia was mild to moderate, with no necrosis or widespread mitotic activity identified. The epithelioid cells were strongly and diffusely positive for cluster of differentiation 34 (CD34) (Fig. 4D), CD31 and transcription factor E3 (TFE3), showed weak staining for CD68 and were negative for pancytokeratin, CD1a, S-100 and sex-determining region Y-box 10 (SOX-10). Proliferation index as measured by Ki-67 was low $(<1 \%)$. Ancillary fluorescence in situ hybridization (FISH) testing for TFE3 rearrangement (Xp11.2) was attempted but was non-informative, with no hybridization signals available for analysis. The hybridization probe failure was felt to be most likely secondary to decalcification of the biopsy material. This constellation of findings was diagnostic of epithelioid hemangioendothelioma (EHE).

Once the diagnosis of EHE was made, a technetium-99m methyl disphosphonate whole-body bone scan was obtained for staging purposes and showed diffuse uptake in the left distal tibia, ankle, and foot with otherwise normal physiologic radiotracer distribution throughout the rest of the body (Fig. 2A and 2B). To evaluate for visceral involvement, computed tomography (CT) of the chest, abdomen, and pelvis showed scattered solid pulmonary nodules measuring less than $5 \mathrm{~mm}$ which remained stable on repeat imaging (Fig. 5A and 5B). Otherwise, there were no CT findings to suggest metastatic disease in the chest, abdomen, or pelvis.

Given the multiple bone involvement isolated to the left foot and ankle, a below the knee amputation was performed. The subsequent specimen demonstrated multicentric skeletal distribution on gross examination, with tumor identified within the distal tibia, tarsal and metatarsal bones. The tumor was present within both the medullary and cortical compartments, with variable involvement of epiphyses, metaphyses and diaphyses. The largest individual focus of tumor was located within the distal tibia and measured up to $3.5 \mathrm{~cm}$. Bone and soft tissue margins were clear. The microscopic features were similar to those seen in the biopsy specimen. Based on the tumor size and multicentric involvement, the tumor was staged as pT1(m) (AJCC 8th edition).
The patient has been doing well since the amputation and wears a leg prosthetic. He continues to work diligently with physical therapy to become more comfortable with his prosthetic and to continue to build his strength. He reports an occasional phantom limb sensation, but denies any phantom limb pain. Thus far, no metastatic disease has been identified; however, he will continue to have routine screening with CT chest, abdomen, and pelvis.

\section{DISCUSSION}

\section{Etiology \& Demographics:}

Epithelioid hemangioendothelioma was first described by Weiss and Enzinger in 1982 as a unique tumor of vascular lineage characterized by an "epithelioid" or "histiocytoid" endothelial cell morphology with a clinical course intermediate between that of hemangioma and angiosarcoma [1,2]. Even though this rare endothelial vascular neoplasm was originally described in the lungs, liver, and other soft tissues, it has increasingly been documented in bone. The prevalence of EHE is less than 1 in 1 million with the most common affected organs being the liver alone (21\%), liver plus lungs (18\%), lung alone (12\%), and bone alone (14\%) [3]. While primary malignant vascular tumors of the bone are extraordinarily rare, representing $<1 \%$ of primary malignant bone tumors, EHE is the most common malignant vascular tumor of bone [1,4-5]. The World Health Organization (WHO) classification of vascular tumors of bone describes EHE as an intermediategrade tumor, separating its classification from the other primary vascular bone tumors: hemangioma (benign), epithelioid hemangioma (locally aggressive), and angiosarcoma (malignant) [4].

EHE occurs more often in males with a 2:1 ratio and with a peak incidence in the 2 nd and 3 rd decades of life, although it can present at any age [1,6-9]. EHE of the bone can affect the calvarium, axial skeleton or appendicular skeleton with the majority of cases $(62 \%)$ affecting the lower extremities [1,9]. It can be a solitary lesion; however, it frequently presents in a multicentric pattern involving a specific region of the body $[1,10]$. The most common clinical presentation of EHE is localized pain and swelling; additionally, pathologic fractures may occur.

\section{Clinical \& Imaging findings:}

The radiographic appearance of EHE typically shows lytic lesions with a variable degree of peripheral sclerosis and an occasional honeycomb appearance [1,10]. Additionally, cortical destruction and expansion may commonly be seen. On MRI, EHE will demonstrate low to intermediate signal intensity on T1-weighted images and high signal intensity on T2weighted images. Due to the high vascularity of the lesion, it will appear homogenously enhanced with contrast on CT and MRI [9]. Bone scintigraphy and fluorodeoxyglucose-positron emission tomography (18F-FDG PET) will show increased uptake of the lesions and can be used to evaluate the extent of the lesions [1,7-8,11-12].

The histologic diagnosis of epithelioid vascular tumors has historically been challenging due to significant overlap between 
the various tumor subtypes [13]. EHE is characterized by sheets or cords of epithelioid cells typically present in a myxohyaline background [1,14-15]. In contrast to angiosarcoma, the epithelioid cells of EHE demonstrate only mild to moderate cytologic atypia and rare mitoses. The vascular origin of the neoplasm is confirmed on immunohistochemistry, which demonstrates staining for vascular markers such as CD31, CD34, friend leukemia integration 1 transcription factor (FLI1) and erythroblast transformation specific-related gene (ERG). Ancillary molecular studies may be useful in confirming the diagnosis, as most cases demonstrate a recurrent $\mathrm{t}(1,3)(\mathrm{p} 36 ; \mathrm{q} 25)$ translocation, resulting in fusion of $\mathrm{WW}$ domain containing transcription regulator 1 (WWTR1) at 3q25 to calmodulin binding transcription activator 1 (CAMTA1) at 1 p36 [3,13,15-17]. Rearrangement of TFE3 at Xp11.2 has been recently described in a subset of EHE's lacking WWTR1CAMTA1 gene fusion, with the most common fusion partner being yes-associated protein 1 (YAP1) at 11q22 [18]. Cases with TFE3 rearrangement show similar morphologic features to conventional EHE, although more pronounced vasoformative features may be noted. Strong nuclear staining for TFE3 on immunohistochemical studies correlates with TFE3 gene rearrangement and is not seen in conventional EHE with WWTR1-CAMTA1 gene fusion [18].

The TFE3 gene is involved in cell growth and proliferation, and rearrangement of the gene results in functional overexpression and oncogenesis. In addition to EHE, TFE overexpression is seen in a histologically diverse array of malignancies, including micropthalmia-associated transcription factor (MiT) family translocation renal cell carcinomas, perivascular epithelioid cell tumor (PEComa), and alveolar soft parts sarcoma [3,19]. Given the oncogenic properties of TFE3, preclinical studies are underway to identify potential therapeutic pharmacologic targets involving the TFE3 growth pathway [20].

\section{Treatment \& Prognosis}

Wide surgical excision is the current treatment of choice for EHE. The local recurrence rate is 13\% [14-15]; therefore, chemotherapy, radiation therapy and embolization are additional treatment options that may be performed with or without surgical excision [1,7-8,14]. However, given the risk of radiation-induced sarcomas, radiation therapy should be reserved for those lesions that are not amenable to wide surgical excision or when lesions are in difficult locations to surgically excise [15].

In conclusion, EHE is a rare, intermediate-grade vascular tumor of bone. It is predominantly seen in males in their 2 nd and 3rd decades of life presenting with localized pain and swelling. Radiographically it appears as lytic lesions with variable sclerotic margins, possible expansion and is often multicentric. The most definitive pathologic characteristic for the diagnosis of EHE is the identification of the translocation $\mathrm{t}(1 ; 3)(\mathrm{p} 36 ; \mathrm{q} 25)$ which fuses WWTR1 to CAMTA1. A subset of WWTR1-CAMTA1 negative EHE's may demonstrate rearrangement of TFE3 with overexpression of TFE3 protein on immunohistochemical studies. Wide surgical excision is currently the treatment of choice.

\section{Differential Diagnoses:}

\section{Sarcoidosis}

Skeletal sarcoidosis is an uncommon manifestation of sarcoidosis. It typically presents with polyostotic involvement of the hand and feet phalanges. Radiographic features include lucent areas with a lacelike, honeycomb appearance. On MRI, the lesions demonstrate hypointense signal on T1- weighted images, hyperintense signal on T2-weighted images, and contrast enhancement. The key to diagnosing skeletal sarcoidosis is identifying additional features of sarcoidosis particularly in the lungs and mediastinum.

\section{Hyperparathyroidism}

Hyperparathyroidism leads to increased levels of parathyroid hormone resulting from either a primary (parathyroid adenoma or parathyroid hyperplasia) or secondary (renal osteodystrophy) cause. The features of hyperparathyroidism predominantly effect the skeletal system leading to subperiosteal resorption involving the radial aspects of the $2 \mathrm{nd} / 3 \mathrm{rd}$ proximal/middle phalanges, subchondral resorption of the distal clavicles, lytic lesions called brown tumors, sclerotic endplates of multiple vertebral bodies (rugger jersey spine), and a salt and pepper skull appearance. Brown tumors on MRI will demonstrate solid and cystic components. The solid components will have low to intermediate signal intensity on $\mathrm{T} 1$ and $\mathrm{T} 2$-weighted imaging with associated contrast enhancement. The cystic components will be high signal intensity on T2-weighted imaging and may show fluidfluid levels.

\section{Enchondromas}

Enchondromas are benign bone tumors that share features with low-grade chondral tumors. There is a variable appearance depending on the location of the enchondroma. In the hands and feet, enchondromas appear as expansile lucent lesions. In the remaining osseous structures, enchondromas have a chondroid matrix with typical "rings and arcs" calcifications. On MRI, enchondromas demonstrate low to intermediate signal intensity on T1-weighted images, high signal intensity on T2weighted images, and variable contrast enhancement. There will be internal foci of low signal intensity on both T1 and T2weighted images that correlate with the chondroid matrix.

\section{Intraosseous Hemangiomas}

Intraosseous hemangiomas are benign vascular hamartomas within the bone that are most frequently seen in the vertebrae. On radiographs, they demonstrate a prominent trabecular pattern with vertical trabeculae seen in the vertebral bodies (corduroy sign). On CT, they have a classic polka-dot appearance in the axial plane and corduroy appearance on the coronal and sagittal planes. The MRI appearance will demonstrate high signal intensity on both T1 and T2-weighted images with associated contrast enhancement.

\section{TEACHING POINT}

Epithelioid hemangioendothelioma of the bone demonstrates a coarsened trabecular appearance radiographically; whereas, more advanced imaging with CT, MRI, and 99mTc-MDP bone scintigraphy will demonstrate a marrow replacing process. Biopsy is ultimately required to make the definitive diagnosis 
and subsequent screening for metastatic disease with CT chest, abdomen, and pelvis must be obtained to look for potential visceral involvement. While this case report presents the typical features of EHE, an exceedingly rare diagnosis, it is an important case for the radiology community to recognize as more advanced imaging, biopsy, and ultimately wide surgical excision are ultimately warranted.

\section{REFERENCES \\ 1. Weissferdt A, Moran CA. Epithelioid} Hemangioendothelioma of the Bone: A Review and Update. Advances in Anatomic Pathology. 2014;21(4):254-259. PMID: 24911250

2. Weiss SW, Enzinger FM. Epithelioid hemangioendothelioma: a vascular tumor often mistaken for carcinoma. Cancer. 1982;50:970-981. PMID: 7093931.

3. Lau K, Massad M, Pollak C, et al. Clinical patterns and outcome in epithelioid hemangioendothelioma with or without pulmonary involvement: Insights from an internet registry in the study of a rare cancer. Chest. 2011;140(5):1312-1318. PMID: 21546438.

4. Fletcher CDM, Bridge JA, Hogendoorn PCW, Mertens F. WHO Classification of tumours of the Soft Tissue and Bone. Lyon, France: IARC Press; 2013:332-338.

5. Bisbinas I, Karabouta Z, Georgiannos D, Lampridis V, Badekas A. Multifocal Epithelioid Hemangioendothelioma of the Foot and Ankle: A Case Report. Journal of Orthopaedic Surgery. 2014;22(1):122-125. PMID: 24781630.

6. Maruyama N, Kumagai Y, Ishida Y, et al. Epithelioid haemangioendothelioma of the bone tissue. Virchows Archiv. A, Pathological Anatomy and Histopathology. 1985;407:159165. PMID: 3927581.

7. Tsuneyoshi M, Dorfman HD, Bauer TW. Epithelioid hemangioendothelioma of bone: A clinicopathologic, ultrastructural, and immunohistochemical study. The American Journal of Surgical Pathology. 1986;10:754-764. PMID: 2430475 .

8. Kleer CG, Unni KK, McLeod RA. Epithelioid hemangioendothelioma of bone. The American Journal of Surgical Pathology. 1996;20:1301-1311. PMID: 8898834.

9. Boutin RD, Spaeth HJ, Mangalik A, Sell JJ. Epithelioid Hemangioendothelioma of Bone. Skeletal Radiology.1996;25(4):391-395. PMID: 8738008.

10. Evans HL, Raymond AK, Ayala AG. Vascular Tumors of Bone: A Study of 17 Cases Other than Ordinary Hemangioma, with an Evaluation of the Relationship of Hemangioendothelioma of Bone to Epithelioid Hemangioma, Epithelioid Hemangioendothelioma, and High-Grade Angiosarcoma. Human Pathology. 2003;34(7):680-689. PMID: 12874764.
11. Hubaut MA, Jaillard A, Eloy C, Petyt G. 18F-FDG PET and Bone Scintigraphy of Epithelioid Hemangioendothelioma. Clinical Nuclear Medicine. 2019;44(2):127-129. PMID: 30516676 .

12. Wenger DE, Wold LE. Malignant vascular lesions of bone: radiologic and pathologic features. Skeletal Radiology. 2000;29(11):619-631. PMID: 11201031.

13. Errani C, Zhang L, Shao SY, et al. A Novel WWTR1CAMTA1 Gene Fusion is a Consistent Abnormality in Epithelioid Hemangioendothelioma of Different Anatomic Sites. Gene Chromosomes Cancer. 2011;50(8):644-653. PMID: 21584898.

14. Weiss SW, Ishak KG, Dail DH, Sweet DE, Enzinger FM. Epithelioid hemangioendothelioma and related lesions. Seminars in Diagnostic Pathology. 1986;3:259-287. PMID: 3303234

15. Angelini A, Mavrogenis AF, Gambarotti M, Merlino B, Picci P, Ruggieri P. Surgical Treatment and Results of 62 Patients with Epithelioid Hemangioendothelioma of Bone. Journal of Surgical Oncology. 2014;109:791-797. PMID: 24643837.

16. Errani C, Vanel D, Gambarotti M, Alberghini M, Picci P, Faldini C. Vascular bone tumors: a proposal of a classification based on clinicopathological, radiographic and genetic features. Skeletal Radiology. 2012;41(12):1495-1507. PMID: 22993209.

17. Tanas MR, Sboner A, Oliveira AM, et al. Identification of a disease defining gene fusion in epithelioid hemangioendothelioma. Science Translational Medicine. 2011;3:98ra82. PMID: 21885404.

18. Antonescu CR, Le Loarer F, Mosquera JM, et al. Novel YAP1-TFE3 fusion defines a distinct subset of epithelioid hemangioendothelioma. Genes Chromosomes Cancer. 2013;52:775-84. PMID: 23737213.

19. Moch H, Cubilla, AL, Humphrey PA, Ulbright TM, Reuter VE, eds. WHO Classification of Tumours of the Urinary System and Male Genital Organs. Lyon, France: IARC Press; 2016:33-34. PMID: 26935559.

20. Damayanti NP, Budka JA, Khella HWZ, et al. Therapeutic Targeting of TFE3/IRS-1/PI3K/mTOR Axis in Translocation Renal Cell Carcinoma. Clin Cancer Res. 2018;24:5977-5989. PMID: 30061365. 


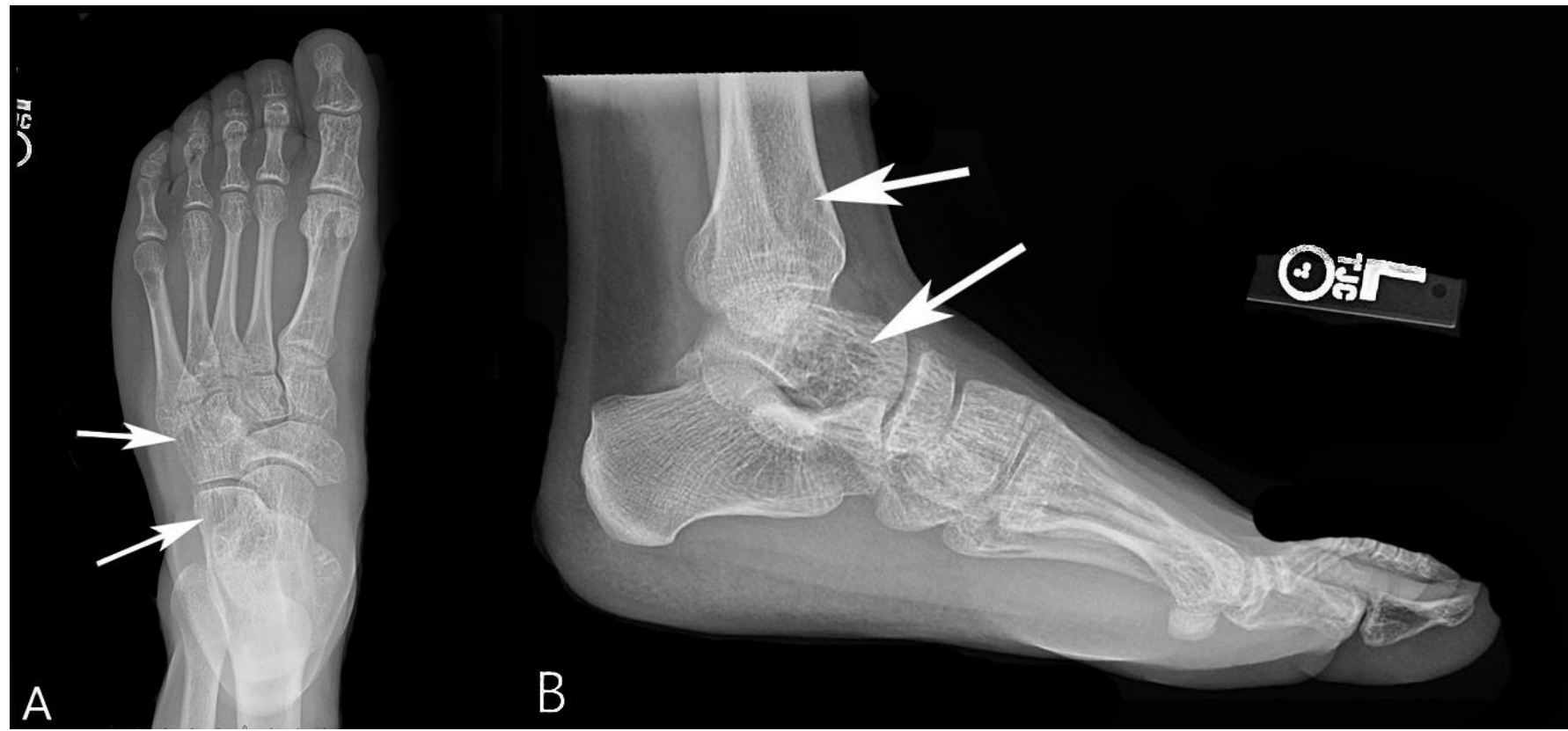

Figure 1: 18-year-old male with epithelioid hemangioendothelioma in the left foot and ankle.

FINDINGS: AP and lateral radiographs of the foot demonstrate a diffuse "lacelike" appearance of medullary bone (arrows) with mixed areas of prominent trabeculation interspersed with lucent areas involving the distal tibia and the bones of the foot.

TECHNIQUE: AP (A) and lateral (B) radiographs of the left foot with settings at a kVp of 60-65 and mAs of 2-3.

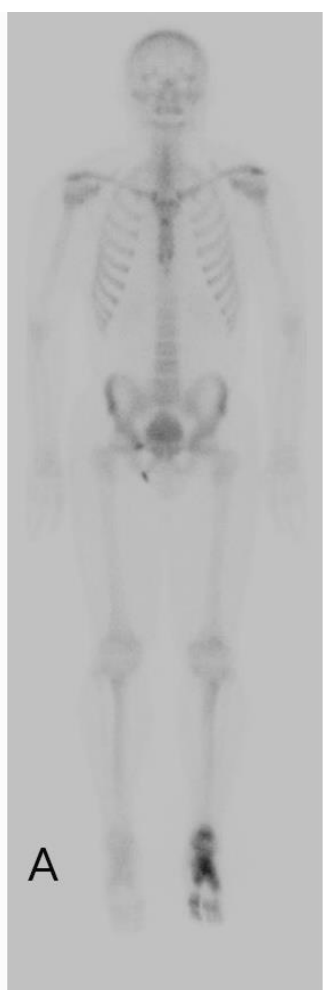

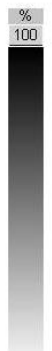

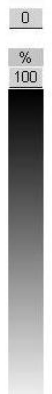

B

RTMED-UT Alpha: $30 \%$

RT ANT LT Alpha: $30 \%$

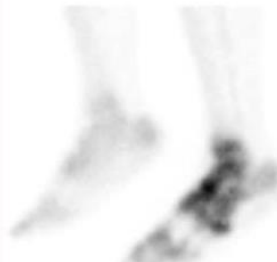

1 of 1

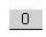
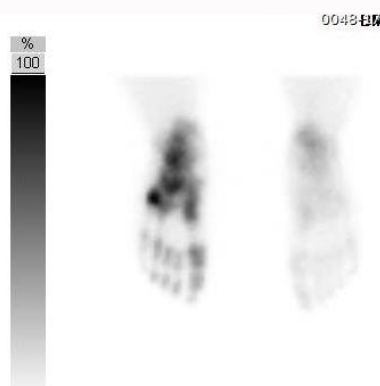

LT POST RT Alpha: $30 \%$

0

LTMED-RLT Alpha:30\%

Figure 2: 18-year-old male with epithelioid hemangioendothelioma in the left foot and ankle.

FINDINGS: Nuclear medicine bone scan demonstrates diffuse radiotracer uptake throughout the distal left tibia, ankle, and foot.

TECHNIQUE: Nuclear medicine bone scan of the whole body and lower extremities after intravenous administration of $25 \mathrm{mCi}$ of Tc-99m methyl disphosphonate. 


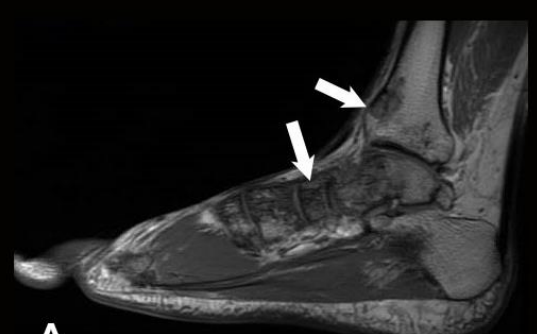

A

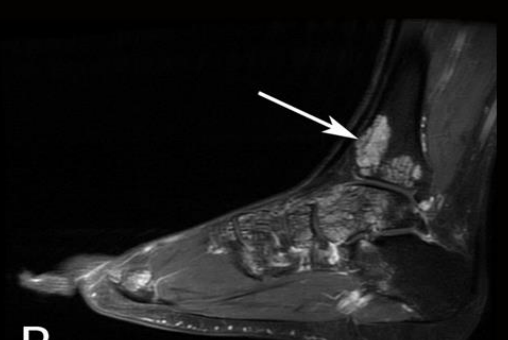

B

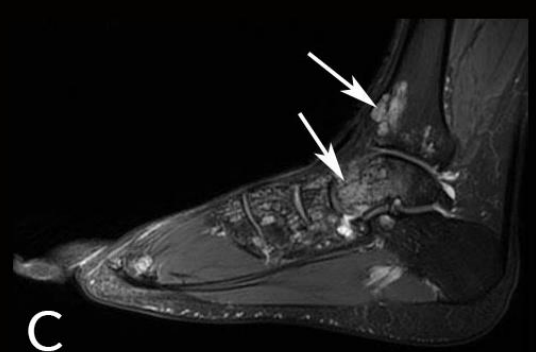

C

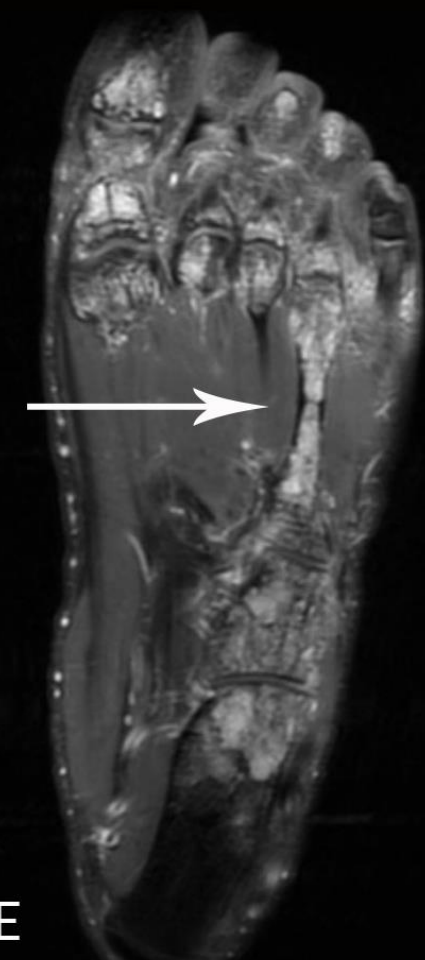

Figure 3: 18-year-old male with epithelioid hemangioendothelioma in the left foot and ankle.

\section{FINDINGS:}

(A) Sagittal T1-weighted MRI of the left foot and ankle demonstrating widespread, polyostotic marrow replacing lesions (arrows) that are isointense to muscle.

(B) Sagittal Proton Density Fat-Saturated MRI of the left foot and ankle demonstrating widespread, polyostotic marrow replacing lesions (arrow) that are slightly hyperintense to muscle.

(C) Sagittal contrast enhanced T1 fat-saturated MRI of the left foot and ankle demonstrating uniform enhancement of the widespread, polyostotic marrow replacing lesions (arrows).

(D) Coronal T2 fat-saturated MRI of the left foot and ankle demonstrating widespread, polyostotic marrow replacing lesions that are slightly hyperintense to muscle.

(E) Axial contrast enhanced T1 fat-saturated MRI of the left foot and ankle demonstrating uniform enhancement of the widespread, polyostotic marrow replacing lesions.

\section{TECHNIQUE:}

(A) Pre-contrast sagittal T1-weighted MRI of the left foot and ankle on a 3 tesla magnet with 3 mm slice thickness.

(B) Pre-contrast sagittal Proton Density Fat-Saturated MRI of the left foot and ankle on a 3 tesla magnet with $3 \mathrm{~mm}$ slice thickness.

(C) Sagittal contrast enhanced T1 fat-saturated MRI of the left foot and ankle on a 3 tesla magnet with 3 mm slice thickness. 16

$\mathrm{mL}$ of Multihance was given.

(D) Pre-contrast coronal T2 fat-saturated MRI of the left foot and ankle on a 3 tesla magnet with 3 mm slice thickness.

(E) Axial contrast enhanced T1 fat-saturated MRI of the left foot and ankle on a 3 tesla magnet with $3 \mathrm{~mm}$ slice thickness. $16 \mathrm{~mL}$ of Multihance was given. 


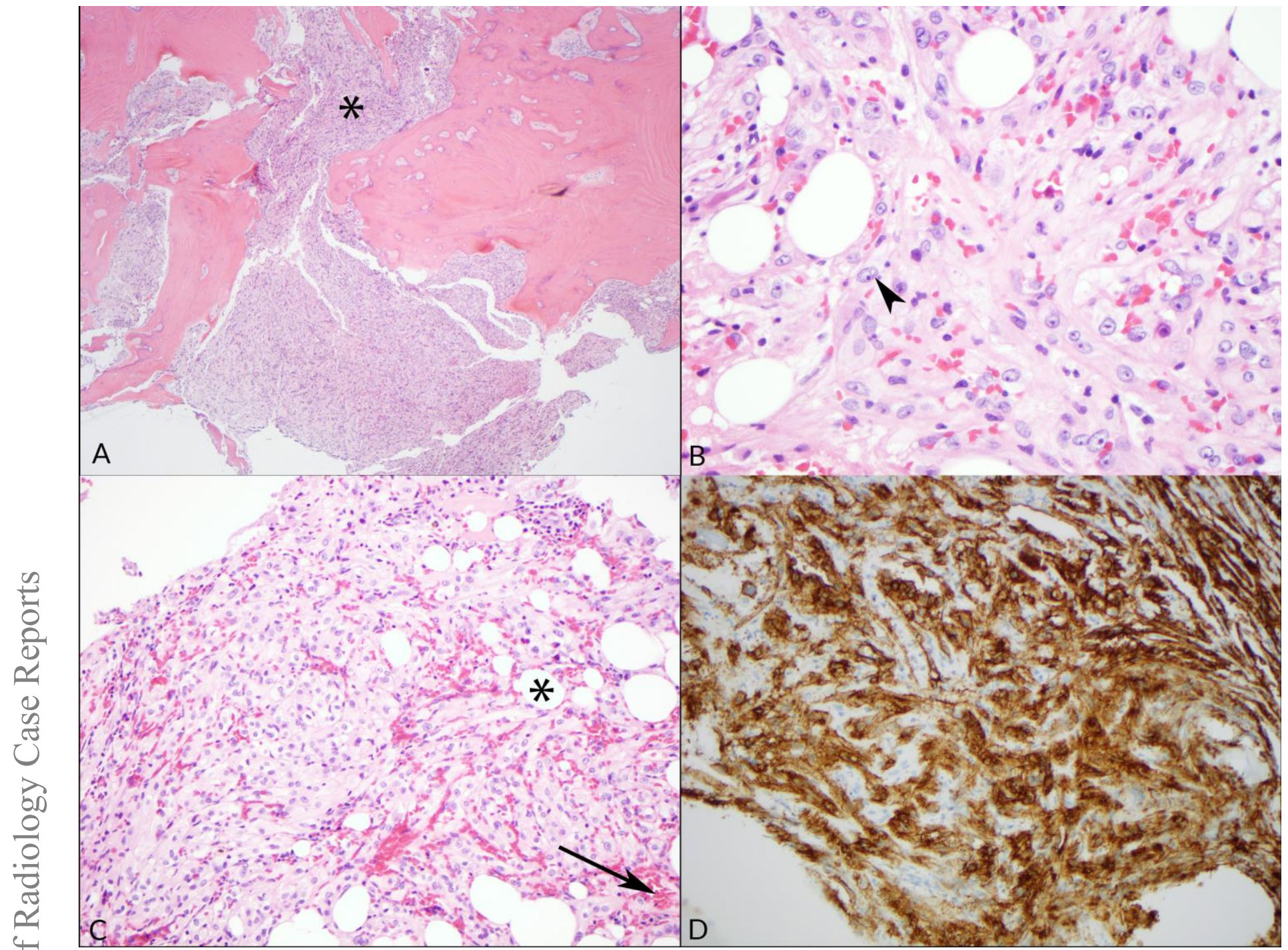

Figure 4: 18-year-old male with epithelioid hemangioendothelioma in the left foot and ankle.

\section{FINDINGS:}

(A) Histologic slide showing sheets of large epithelioid cells (asterisk) infiltrating the marrow space (H\&E, 40X).

(B and C) Histologic slides showing sheets of epithelioid cells with abundant eosinophilic cytoplasm, oval to kidney-shaped nuclei (arrowhead) and fine chromatin. Occasional cytoplasmic vacuolization (asterisk) is noted, with some of the vacuoles filled with red blood cells (arrow) (H\&E, 200X and 400X).

(F) Histologic slide showing strong and diffuse staining for CD34 (200X). 


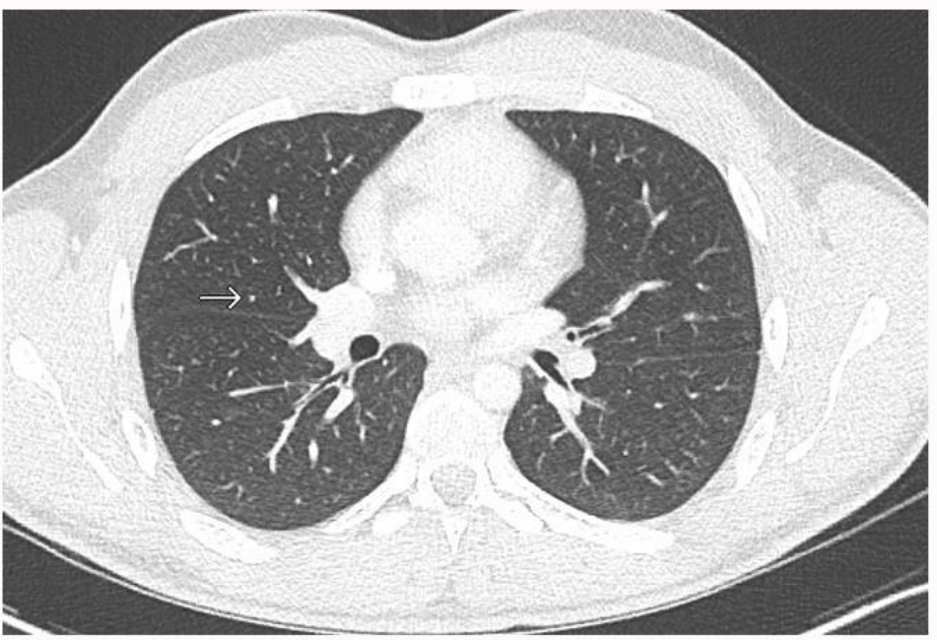

A

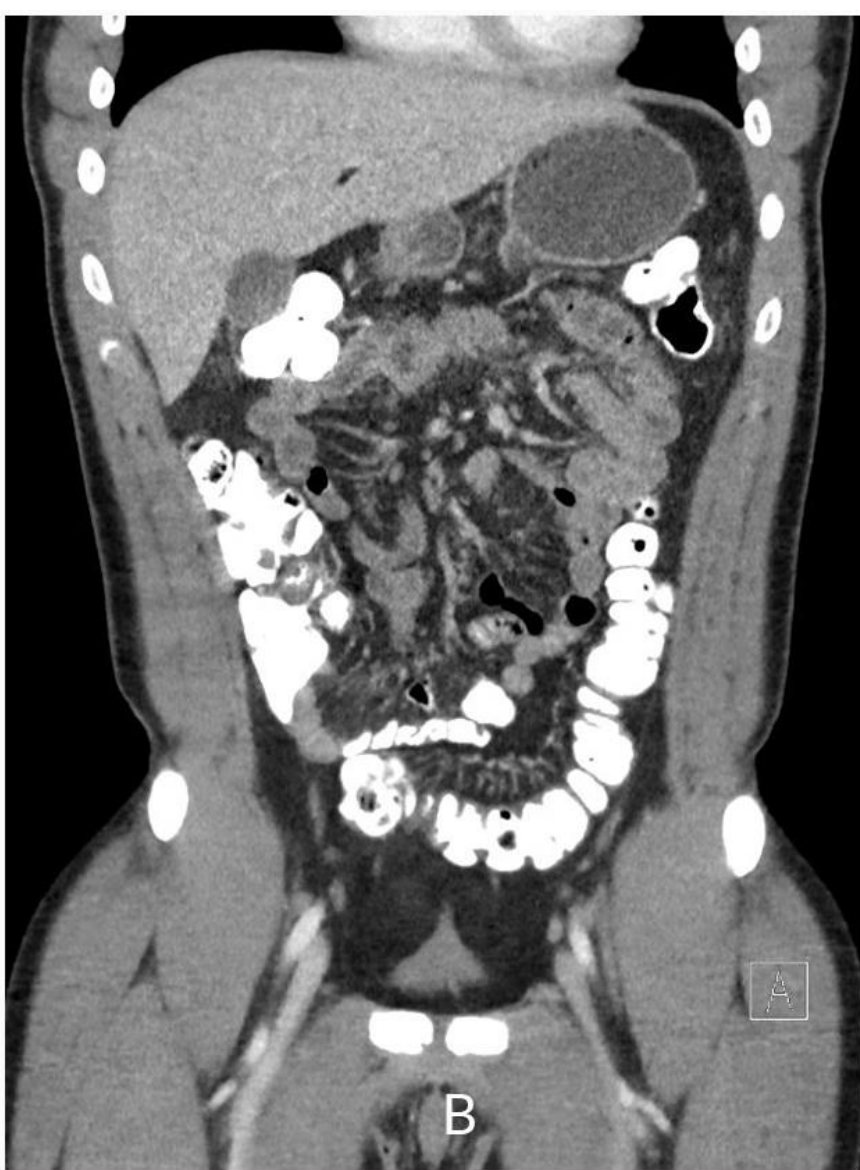

Figure 5: 18-year-old male with epithelioid hemangioendothelioma in the left foot and ankle.

FINDINGS: Axial CT of the chest demonstrates a sub-6mm solid micronodule. Coronal CT of the abdomen demonstrates no evidence of metastatic disease.

\section{TECHNIQUE:}

(A) Post-contrast axial CT of the chest with $1 \mathrm{~mm}$ slice thickness on a Siemens SOMATOM Definition Flash helical CT scanner. $100 \mathrm{~mL}$ of Omnipaque 350 intravenous contrast and $1 \mathrm{~L}$ of dilute Omnipaque 240 oral contrast was given. Settings were set at a $\mathrm{kVp}$ of 100 and $\mathrm{mAs}$ of 160 . Total DLP was $492 \mathrm{mGycm}$ for the combined chest, abdomen, and pelvis.

(B) Post-contrast coronal CT of the abdomen and pelvis with $5 \mathrm{~mm}$ slice thickness on a Siemens SOMATOM Definition Flash helical CT scanner. $100 \mathrm{~mL}$ of Omnipaque 350 intravenous contrast and $1 \mathrm{~L}$ of dilute Omnipaque 240 oral contrast was given. Settings were set at a kVp of 100 and mAs of 142. Total DLP was $492 \mathrm{mGycm}$ for the combined chest, abdomen, and pelvis.

\begin{tabular}{|l|l|}
\hline Etiology & $\begin{array}{l}\text { Intermediate-grade tumor of vascular lineage characterized by an "epithelioid" or "histiocytoid" } \\
\text { endothelial cell morphology }\end{array}$ \\
\hline Incidence & $\begin{array}{l}\text { Most common malignant vascular tumor of bone; however, primary malignant vascular tumors of the } \\
\text { bone are rare, representing <1\% of primary malignant bone tumors }\end{array}$ \\
\hline Gender ratio & Male preponderance with a 2:1 ratio \\
\hline Age predilection & Peak incidence in the 2nd and 3rd decades of life \\
\hline Risk factors & No known risk factors \\
\hline Treatment & $\begin{array}{l}\text { Wide surgical excision is the treatment of choice. Chemotherapy, radiation therapy and embolization } \\
\text { are additional treatment options that may be performed with or without surgical excision. }\end{array}$ \\
\hline Prognosis & $13 \%$ local recurrence rate \\
\hline findings on imaging & $\begin{array}{l}\text { Radiographs and CT: Lytic lesions with variable sclerotic margins, cortical destruction and expansion, } \\
\text { and homogeneous enhancement. } \\
\text { MRI: Marrow replacing lesions that have low to intermediate T1 signal intensity, high T2 signal } \\
\text { intensity, and homogenous contrast enhancement }\end{array}$ \\
\hline
\end{tabular}

Table 1: Summary table of epithelioid hemangioendothelioma. 


\begin{tabular}{|c|c|c|}
\hline & Radiograph/CT features & MRI features \\
\hline $\begin{array}{l}\text { Epithelioid } \\
\text { hemangioendothelioma }\end{array}$ & $\begin{array}{l}\text { - Lytic lesions with variable degrees of } \\
\text { peripheral sclerosis } \\
\text { - Cortical destruction and expansion may } \\
\text { commonly be seen } \\
\text { - Homogeneous enhancement on contrast } \\
\text { enhanced CT }\end{array}$ & $\begin{array}{l}\text { - Low to intermediate T1 signal intensity } \\
\text { - High T2 signal intensity } \\
\text { - Homogenous contrast enhancement }\end{array}$ \\
\hline Sarcoidosis & $\begin{array}{l}\text { - Lucent areas with a lacelike honeycomb } \\
\text { appearance involving the hands and feet }\end{array}$ & $\begin{array}{l}\text { - Low T1 signal intensity } \\
\text { - High T2 signal intensity } \\
\text { - Contrast enhancement }\end{array}$ \\
\hline Hyperparathyroidism & $\begin{array}{l}\text { - Subperiosteal resorption of the radial aspects } \\
\text { of the } 2 \text { nd /3rd proximal/middle phalanges } \\
\text { - Subchondral resorption of the distal clavicles } \\
\text { - Brown tumors } \\
\text { - Rugger jersey spine } \\
\text { - Salt \& pepper skull }\end{array}$ & $\begin{array}{l}\text { - Brown tumors demonstrate solid and } \\
\text { cystic components } \\
\text { - Solid components will have low to } \\
\text { intermediate T1 and T2 signal intensity } \\
\text { with contrast enhancement } \\
\text { - Cystic components will have high T2 } \\
\text { signal intensity and may show fluid-fluid } \\
\text { levels }\end{array}$ \\
\hline Enchondromas & $\begin{array}{l}\text { - Hands and feet: expansile lucent lesions } \\
\text { - Remaining osseous structures: chondroid } \\
\text { matrix with typical "rings and arcs" } \\
\text { calcifications }\end{array}$ & $\begin{array}{l}\text { - Low to intermediate T1 signal intensity } \\
\text { - High T2 signal intensity } \\
\text { - Variable contrast enhancement } \\
\text { - Internal foci of low T1 and T2 signal } \\
\text { intensity correlating with chondroid } \\
\text { matrix }\end{array}$ \\
\hline intraosseous hemangiomas & $\begin{array}{l}\text { - Radiographs: Prominent trabecular pattern } \\
\text { with vertical trabeculae seen in the vertebral } \\
\text { bodies } \\
\text { - CT: Classic polka-dot appearance in the axial } \\
\text { plane and corduroy appearance on the coronal } \\
\text { and sagittal planes }\end{array}$ & $\begin{array}{l}\text { - MRI: High T1 and T2 signal intensity } \\
\text { with associated contrast enhancement }\end{array}$ \\
\hline
\end{tabular}

Table 2: Differential diagnosis table for epithelioid hemangioendothelioma.

\section{ABBREVIATIONS}

CAMTA1 $=$ calmodulin binding transcription activator 1

$\mathrm{CD}=$ cluster of differentiation

$\mathrm{CT}=$ computed tomography

18F-FDG PET = fluorodeoxyglucose-positron emission tomography

$\mathrm{EHE}=$ epithelioid hemangioendothelioma

ERG $=$ erythroblast transformation specific-related gene

$\mathrm{FISH}=$ fluorescence in situ hybridization

FLI-1 = friend leukemia integration 1 transcription factor

MiT $=$ micropthalmia-associated transcription factor

MRI = magnetic resonance imaging

PEComa $=$ perivascular epithelioid cell tumor

SOX10 $=$ sex-determining region Y-box 10

TFE3 = transcription factor E3

$\mathrm{WHO}=$ world health organization

WWTR $1=\mathrm{WW}$ domain containing transcription regulator 1

YAP1 $=$ yes-associated protein 1

\section{Online access}

This publication is online available at: www.radiologycases.com/index.php/radiologycases/article/view/4052

\section{Peer discussion}

Discuss this manuscript in our protected discussion forum at: www.radiolopolis.com/forums/JRCR

\section{Interactivity}

This publication is available as an interactive article with scroll, window/level, magnify and more features.

Available online at www.RadiologyCases.com

\section{Published by EduRad}

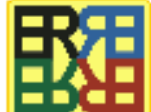

www.EduRad.org

\section{KEYWORDS}

Epithelioid hemangioendothelioma; vascular tumor; osseous tumor; lower extremity; polyostotic bone tumor 